\title{
A case for assessment of oscillatory breathing during cardiopulmonary exercise test in risk stratification of elderly patients with chronic heart failure
}

\author{
Angela Beatrice Scardovi ${ }^{\text {a, } *}$, Renata De Maria ${ }^{b}$, Alessandro Ferraironi ${ }^{a}$, Laura Gatto ${ }^{c}$, Andrea Celestini ${ }^{a}$, \\ Silvia Forte ${ }^{\mathrm{d}}$, Marina Parolini ${ }^{\mathrm{b}}$, Sebastiano Sciarretta ${ }^{\mathrm{e}}$, Roberto Ricci ${ }^{\mathrm{a}}$, Marco Guazzi ${ }^{\mathrm{f}}$ \\ a Cardiology, S. Spirito Hospital, Rome, Italy \\ b CNR Clinical Physiology Institute, Milan, Italy \\ c Cardiology, San Giovanni-Addolorata Hopital, Rome, Italy \\ d Pneumology, San Giovanni-Addolorata Hospital, Rome, Italy \\ e Cardiology, S. Andrea Hospital, University of Medicine "La Sapienza", Rome, Italy \\ ${ }^{\mathrm{f}}$ Cardiopulmonary Laboratory, University of Milan, San Paolo Hospital, Milan, Italy
}

\section{A R T I C L E I N F O}

\section{Article history:}

Received 10 January 2011

Accepted 10 February 2011

Available online $\mathrm{xxxx}$

\section{Keywords:}

Elderly

Heart failure

Cardiopulmonary exercise test

Ventilatory inefficiency

\begin{abstract}
A B S T R A C T
The prognostic value of exercise oscillatory breathing (EOB) during cardiopulmonary test (CPX) has been described in young chronic heart failure (HF) patients. We assessed the prognostic role of EOB vs other clinical and ventilatory parameters in elderly HF patients performing a maximal CPX.

Methods and results: We prospectively followed-up $370 \mathrm{HF}$ outpatients $\geq 65$ years after a symptom limited $\mathrm{CPX}$. We tested the predictive value of clinical and ventilatory parameters for all-cause mortality and a composite of all-cause mortality and HF hospitalizations. Median age was 74 years, $51 \%$ had ischemic heart disease, 25\% NYHA class III; ejection fraction was 41\% [34-50]. Peak oxygen consumption $\left(\mathrm{PVO}_{2}\right)$ was 11.9 [9.9-14] $\mathrm{mL} / \mathrm{kg} / \mathrm{min}$, the slope of the regression line relating ventilation to $\mathrm{CO}_{2}$ output, $\left(\mathrm{VE} / \mathrm{VCO}_{2}\right.$ slope $)$ was 33.9 [29.8-39.2]. EOB was found in 58\% of patients. At follow-up, 84 patients died and overall 158, using a time-to-first event approach, met the composite end-point. Independent predictors of all-cause mortality were $\mathrm{CPX} \mathrm{EOB}$ and the ratio of $\mathrm{VE} / \mathrm{VCO}_{2}$ slope to peak $\mathrm{VO}_{2}$, hemoglobin, creatinine and body mass index. The area under the ROC curve (AUC) of the Cox multivariable model was 0.80 (95\% CI 0.73 to 0.87). Independent predictors of the composite end-point were $\mathrm{EOB}, \mathrm{VE} / \mathrm{VCO}_{2}$ slope, hemoglobin and $\mathrm{HF}$ admissions in the previous year (Model AUC 0.75) (95\% CI 0.69 to 0.81 ).

Conclusions: Among elderly HF patients, EOB prevalence is higher than middle-aged cohorts. EOB and the ratio of $\mathrm{VE} / \mathrm{VCO}_{2}$ slope to peak $\mathrm{VO}_{2}$ resulted the strongest ventilatory predictor of all-cause mortality, independent of ventricular function.
\end{abstract}

(c) 2011 Elsevier Ireland Ltd. All rights reserved.

\section{Introduction}

Prognostic stratification of patients with chronic heart failure (HF) is useful for follow-up planning and for tailoring therapeutic interventions [1]. The elderly HF population is markedly heterogeneous. Many elderly HF patients are frail with multiple comorbidities and for them the only realistic aim is to relieve symptoms and improve quality of life. However a substantial proportion of these patients, without significant comorbidities or exercise limitations, fully qualify for management and prognostic stratification similar to those routinely used for younger patients [2].

Cardiopulmonary exercise test (CPX) provides simultaneous assessment of the performance of the heart, lungs, muscles and

\footnotetext{
* Corresponding author at: Cardiology Department, S. Spirito Hospital, Lungotevere in Sassia 1, 00143 Rome, Italy. Tel.: + 39 0668352264, +39 3388479212.

E-mail address: ab.scardovi@libero.it (A.B. Scardovi).
}

cellular respiration during physical exercise. Accordingly, CPX has an established role in the identification of patients with exercise intolerance at higher risk for major cardiac events [3]. Although guidelines advocate the test to select heart transplant candidates and to distinguish between respiratory or cardiac limitations [4], CPX use and application are now beyond this role, providing prognostic and functional information in the whole spectrum of HF severity [3,4]. In particular, СPX can be an important risk stratification tool even in elderly HF patients who cannot be considered for heart transplant, provided that they are physically able to perform a maximal exercise test [2]. We previously demonstrated [5] that even in the elderly the test is safe, feasible and reliable in terms of prognostic stratification.

Classification into high, intermediate and low risk may substantially improve delivery of health services, as patients should receive care at the level best suited to their individual needs. Low-risk patients may be routinely managed in primary care, whereas patients at intermediate risk should receive strict follow-up by cardiologists 
and be timely referred for non-pharmacological treatment. In patients at high risk with a poor short term outlook advanced treatment options may be futile, and the focus of care should be on symptom relief.

In a recent study conducted on a range of patients of a suitable age for heart transplantation, ventilatory efficiency assessed by the minute ventilation/carbon dioxide production $\left(\mathrm{VE} / \mathrm{VCO}_{2}\right)$ slope during CPX, allowed to develop a reliable risk stratification system for a wide range of $\mathrm{HF}$ patients with different severity stages [6]. Exercise oscillatory ventilation (EOB), a cyclic breathing pattern of oscillations in minute ventilation and gas exchange kinetics during incremental exercise, has been suggested to represent a stronger predictor than $\mathrm{VE} / \mathrm{VCO}_{2}$ slope [7]. EOB often co-exists with central sleep apnea and may reflect a more severe derangement of the ventilatory control system [8]. Furthermore, recent findings support the usefulness of EOB in predicting death from arrhythmic causes [9].

The relative prognostic role of $\mathrm{VE} / \mathrm{VCO}_{2}$ slope and $\mathrm{EOB}$ has not yet been fully elucidated in the elderly HF population. Our aim was to assess the prognostic value of ventilatory variables for all-cause mortality and a composite of mortality and hospitalization for HF.

\section{Methods}

Elderly ( $\geq 65$ years) patients with a clinical diagnosis of HF [1] consecutively evaluated at the CPX laboratories of Santo Spirito Hospital, Rome, and San Paolo Hospital, Milan, Italy between March 1993 and June 2008 were enrolled in this study. The indication to CPX was clinical decision-making for follow-up planning and tailored medical or electrical treatment. Exclusion criteria were : 1 ) mitral stenosis or previous mitral valve surgery 2 ) severe aortic stenosis (peak systolic velocity $\geq 4.5 \mathrm{~m} / \mathrm{s}$ ) or severe aortic insufficiency 3) significant pulmonary or liver disease 4) recent ( $\leq 3$ months) myocardial infarction 5) New York Heart Association (NYHA) functional class IV 6) clinical limitations to exercise (orthopedic problems, severe peripheral vascular disease, complex arrhythmias, history of recent syncope or unstable angina, high degree atrio-ventricular block, severe anemia, severe hyperthyroidism, and resting blood pressure $\geq 220 / 120 \mathrm{~mm} \mathrm{Hg}$ ) and 7) worsening symptoms or need for intravenous inotropic support during the last 4 weeks.

Patients were in NYHA classes I-III on stable drug doses since at least 3 months and able to perform a CPX test with a respiratory exchange ratio (RER) $>1.00$, indicating the achievement of anaerobic exercise conditions, and with a peak oxygen uptake $\left(\mathrm{PVO}_{2}\right)$ $<25 \mathrm{mg} / \mathrm{kg} / \mathrm{min}$. Among 545 elderly HF patients, 370 were enrolled, while $135(27 \%)$ were excluded according to the inclusion/exclusion criteria and $25(5 \%)$ were excluded for sub-maximal exercise $(\mathrm{RER}<1)$. Baseline laboratory assessment (brain natriuretic peptide (BNP), hemoglobin, serum sodium and serum creatinine) was performed according to standard laboratory methods.

All consecutive subjects who met the entry criteria were enrolled and tracked after $\mathrm{CPX}$. All patients received routine follow-up care at the two participating institutions. Outcome data of patients who did not attend their scheduled appointments $(<10 \%$ of those enrolled) were obtained by telephone interview of the patient or his/her family; none of these patients had been admitted to a hospital. All subjects signed a written informed consent, and institutional review board approval was obtained at each institution.

\subsection{CPX procedure and data collection}

The same equipment and exercise testing protocol was used in both participating centers. Symptom-limited CPX was performed by cycle ergometry ramping, analysis was performed with a metabolic cart (SensorMedics Vmax29, Yorba Linda, Calif). Before each test, the equipment was calibrated in standard fashion with reference gases. Standard 12-lead ECGs were obtained at rest, each minute during exercise, and for at least 5 min during the recovery phase. Blood pressure was measured with a standard cuff sphygmomanometer. Minute ventilation (VE), oxygen uptake $\left(\mathrm{VO}_{2}\right)$, carbon dioxide output $\left(\mathrm{VCO}_{2}\right)$, and other cardiopulmonary variables were acquired on a breath-by-breath basis and averaged over 10-second intervals. $\mathrm{PVO}_{2}$ and peak respiratory exchange ratio was expressed as the highest averaged samples obtained during the exercise test. $\mathrm{VE}$ and $\mathrm{VCO}_{2}$ values were acquired from the initiation of exercise to peak exercise. For the calculation of $\mathrm{VE} / \mathrm{VCO}_{2}$ slope linear relationship, we measured all data points from the beginning to the end of exercise, in agreement with what was recently demonstrated [10]. The ratio of $\mathrm{VE} / \mathrm{VCO}_{2}$ slope to $\mathrm{PVO}_{2}$, previously suggested as a better predictor of prognosis than either variable alone [11] was also analyzed.

EOB was assessed with the criteria previously reported by Leite et al. [12] and defined as 1) 3 or more regular oscillatory fluctuations in VE; 2) minimal average amplitude of ventilatory oscillation of $5 \mathrm{~L}$ (peak value minus the average of two inbetween consecutive nadirs); and 3) a regular oscillation as defined by an SD of 3 consecutive cycle lengths (time between two consecutive nadirs) within $20 \%$ of the average. A RER>1.00 was selected for the definition of maximal exercise.

\subsection{Doppler echocardiography}

Left ventricular ejection fraction (LVEF) was measured according to the modified Simpson's biplane method. Mitral peak early (E) and late (A) wave flow velocity and the E/A ratio were determined by pulsed-wave Doppler echocardiography [13].

\subsection{Statistical analysis}

Categorical variables are presented as frequency percents and continuous variables as median values [interquartile range]. Between-group differences according to EOB were tested by chi-square test and Student's $t$-test or non parametric testing according to normal or non normal variable distribution.

Study end-points were all-cause mortality and a combination of all-cause mortality or hospital admission for worsening HF. To determine the independent predictors of outcome, we entered variables significant by univariable analysis $(p<0.10)$ in multivariable Cox proportional hazards models that identified relevant covariates through a forward selection procedure. Hazard ratios (HR) are presented with 95\% confidence intervals (CI). Non categorical variables were entered as continuous values and HR are expressed as risk per 1 unit change. Model discrimination was tested using the c statistic and expressed as area under the ROC curve (AUC).

A p value $<0.05$ was considered statistically significant. All statistical tests were two-tailed. Data were analyzed using The Statistical Package for the Social Sciences SPSS 10.

\section{Results}

\subsection{Study population}

Clinical characteristics and CPX parameters of the 370 elderly stable HF outpatients included in this study are summarized in Table 1. Patients were studied after optimization of drug therapy betablockers (carvedilol, mean dose $18 \pm 6 \mathrm{mg}$ ) in 227 (61\%), and RASinhibitors in 340 (92\%). Cardiac resynchronization therapy and a cardioverter defibrillator had been implanted in 21 patients (5.6\%).

EOB was found in 215 patients (58\%). EOB was significantly associated to male gender ( $63 \%$ of men and $45 \%$ of women had EOB, $\mathrm{p}<0.001$ ), an HF admission in the previous year ( $66 \%$ of patients with and $46 \%$ of those without a recent HF admission had EOB, $\mathrm{p}<0.001$ ), and a lower median LVEF ( $40 \%$ in patients with vs $45 \%$ in those without EOB, $\mathrm{p}<0.001$ ).

\subsection{Prediction of outcome}

During a median tracking period of 29 [12-47] months, 84 patients died ( 51 of worsening HF, 17 of sudden death, 1 of acute myocardial infarction, 2 of stroke, 1 of pulmonary embolism, 10 of cancer, and 1 of respiratory failure); 90 subjects were admitted for worsening HF. Using a time-to-first event approach, overall 158 patients met the combined end-point of all-cause mortality or HF admission. Variables significantly associated to all-cause mortality and to the combined endpoint by univariable analysis are reported in Tables 2 and 3. By multivariable analysis, EOB predicted both all-cause mortality and the combined end point, independently of ventricular function (Table 4). The adjusted hazard curves for all-cause mortality or the combined end point for patients with or without EOB are shown in Figs. 1 and 2, respectively. Model AUC was 0.80 (95\% CI 0.73 to 0.87 ) for all-cause mortality and 0.75 (95\% CI 0.69 to 0.81 ) for the composite end-point (Figs. 3 and 4).

\section{Discussion}

To the best of our knowledge, this is the first study to specifically assess the incidence of EOB in an elderly HF population, and to describe its relationship with other ventilatory abnormalities during $\mathrm{CPX}$ and its prognostic impact. We found that EOB is much more common in aged HF patients (58\%) than previously reported in younger populations (20-30\%) [7-9,14]. Still, in agreement with previous data $[7-9,14]$ on middle aged HF patients, EOB emerged as the strongest independent CPX predictor of survival even in our elderly series. 
Table 1

Clinical characteristics and main parameters in the 370 study patients.

\begin{tabular}{ll}
\hline Female gender & $107(29 \%)$ \\
Ischemic etiology & $190(51 \%)$ \\
NYHA class III & $92(25 \%)$ \\
Age (years) & $74[71-78]$ \\
BMI & $25.4[23.1-27.7]$ \\
Sodium mmol/L & $140[138-141]$ \\
Creatinine mg/dL & $1.22[1.0-1.5]$ \\
Hemoglobin g/L & $14[13.8-14.1]$ \\
BNP pg/mL & $152[86-400]$ \\
HF admissions in the previous year & $199(54 \%)$ \\
Atrial fibrillation & $51(14 \%)$ \\
Permanent pacemaker & $29(8 \%)$ \\
Left ventricular ejection fraction (\%) & $41[34-50]$ \\
E/A ratio & $0.85[0.61-1.47]$ \\
RER & $1.10[1.02-1.19]$ \\
$\mathrm{Workload}(\mathrm{W})$ & $64[48-81]$ \\
$\mathrm{PVO}_{2}(\mathrm{~mL} / \mathrm{kg} / \mathrm{min})$ & $11.9[9.9-14]$ \\
$\% \mathrm{PVO}_{2}$ & $61[50-75]$ \\
$\mathrm{VE}_{\mathrm{VCO}}$ slope & $33.9[29.8-39.2]$ \\
$\mathrm{VE}_{\mathrm{VCO}}$ slope/PVO \\
$\mathrm{EOB}$ & $2.8[2.19-3.72]$ \\
\hline
\end{tabular}

Data are presented as median [interquartile range] or numbers (\%). A, Transmitral A Velocity; BMI, body mass index; BNP, brain natriuretic peptide; LVEF, left ventricular ejection fraction; E, Transmitral E Velocity; EOB, exercise oscillatory breathing; $\mathrm{PVO}_{2}$, peak oxygen uptake;\% $\mathrm{PVO}_{2}$, percent predicted $\mathrm{PVO}_{2}$; RER, respiratory exchange ratio; $\mathrm{VE}$, ventilation; $\mathrm{VE} / \mathrm{VCO}_{2}$ slope, slope of the regression line relating $\mathrm{VE}$ to carbon dioxide production; and $\mathrm{VO}_{2} \mathrm{AT}$, oxygen uptake at anaerobic threshold.

While the poor prognosis of elderly HF patients is well known [15], few studies have focused on the role of CPX for risk stratification in this specific population or analyzed the prognostic value of ventilatory variables in patients of an age similar to those enrolled in our study [15-17]. Both Davies et al. [15] and Mejhert et al. [16] reported a powerful predictive role of $\mathrm{VE} / \mathrm{VCO}_{2}$ slope in a small series of $\mathrm{HF}$ patients. However, their analyses were restricted to patients with systolic heart failure. In a larger study by Cicoira et al. [17], both $\mathrm{PVO}_{2}$ and $\mathrm{VE} / \mathrm{VCO}_{2}$ slope were mortality predictors. In our previous report [18] in 331 consecutive outpatients aged $>70$ years with mild to moderate congestive HF and both impaired and preserved systolic functions, we found that a reduced glomerular filtration rate and a steeper $\mathrm{VE} / \mathrm{VCO}_{2}$ slope provide additional independent prognostic information across the spectrum of ventricular function impairment and help to identify high risk patients better than BNP and LVEF. In 396 elderly ( $>65$ years) HF patients, Parikh et al. [19] demonstrated that $\mathrm{PVO}_{2}$ and the Heart Failure Survival Score were both excellent

Table 2

Univariable predictors of all cause death.

\begin{tabular}{|c|c|c|c|c|}
\hline & Chi-square & $\mathrm{P}$ & $\mathrm{HR}$ & $95 \% \mathrm{CI}$ \\
\hline Hemoglobin g/dL & 39.538 & $<0.001$ & 0.597 & $0.509-0.699$ \\
\hline $\mathrm{BNP} \mathrm{pg} / \mathrm{mL}$ & 19.88 & $<0.001$ & 1.001 & $1.001-1.001$ \\
\hline $\mathrm{EOB}$ & 14.625 & $<0.001$ & 2.26 & $1.569-4.375$ \\
\hline NYHA class III vs I-II & 11.464 & 0.001 & 2.116 & $1.358-3.298$ \\
\hline $\operatorname{LVEF}(\%)$ & 11.459 & 0.001 & 0.968 & $0.950-0.987$ \\
\hline Creatinine mg/dL & 10.696 & 0.001 & 1.657 & $1.229-2.234$ \\
\hline $\mathrm{VE} / \mathrm{VCO}_{2}$ slope & 10.557 & 0.001 & 1.035 & $1.014-1.057$ \\
\hline Sodium $\mathrm{mmol} / \mathrm{L}$ & 7.922 & 0.005 & 0.93 & $0.885-0.978$ \\
\hline HF admission previous 12 months & 7.844 & $<0.001$ & 2.19 & $1.248-3.844$ \\
\hline E/A ratio & 6.63 & 0.011 & 1.207 & $1.044-1.395$ \\
\hline Body mass index & 6.429 & 0.011 & 0.92 & $0.862-0.981$ \\
\hline Workload (W) & 5.998 & 0.014 & 0.989 & $0.981-0.998$ \\
\hline $\mathrm{VE} / \mathrm{VCO}_{2}$ slope $/ \mathrm{PVO}_{2}$ & 5.605 & 0.019 & 1.128 & $1.020-1.248$ \\
\hline $\mathrm{PVO}_{2}(\mathrm{~mL} / \mathrm{kg} / \mathrm{min})$ & 5.52 & 0.017 & 0.924 & $0.865-0.986$ \\
\hline Age & 4.751 & 0.03 & 1.045 & $1.004-1.087$ \\
\hline Ischemic etiology & 1.786 & 0.183 & 1.348 & $0.869-2.092$ \\
\hline$\% \mathrm{PVO}_{2}$ & 1.006 & 0.314 & 0.994 & $0.983-1.006$ \\
\hline Beta-blockers & 0.241 & 0.624 & 0.897 & $0.582-1.384$ \\
\hline Female gender & 0.091 & 0.764 & 0.928 & $0.569-1.512$ \\
\hline
\end{tabular}

Table 3

Univariable predictors of all cause death and HF admission.

\begin{tabular}{|c|c|c|c|c|}
\hline & Chi-square & $\mathrm{P}$ & HR & $95 \% \mathrm{CI}$ \\
\hline HF admission previous 12 months & 26.883 & $<0.001$ & 2.753 & $1.847-4.105$ \\
\hline Hemoglobin g/dL & 16.672 & $<0.001$ & 0.776 & $0.688-0.876$ \\
\hline $\mathrm{BNP} \mathrm{pg} / \mathrm{mL}$ & 16.183 & $<0.001$ & 1.001 & $1.000-1.001$ \\
\hline EOB & 15.026 & $<0.001$ & 1.945 & $1.381-2.74$ \\
\hline Workload (W) & 11.662 & 0.001 & 0.989 & $0.983-0.995$ \\
\hline NYHA class III vs I-II & 11.301 & 0.001 & 1.788 & $1.268-2.522$ \\
\hline $\mathrm{PVO}_{2}(\mathrm{~mL} / \mathrm{Kg} / \mathrm{min})$ & 9.863 & 0.001 & 0.929 & $0.889-0.972$ \\
\hline $\mathrm{VE} / \mathrm{VCO}_{2}$ slope & 8.426 & 0.004 & 1.04 & $1.008-1.041$ \\
\hline $\mathrm{VE} / \mathrm{VCO}_{2}$ slope $/ \mathrm{PVO}_{2}$ & 6.994 & 0.009 & 1.1 & $1.025-1.182$ \\
\hline $\operatorname{LVEF}(\%)$ & 6.37 & 0.012 & 0.983 & $0.971-0.996$ \\
\hline $\mathrm{E} / \mathrm{A}$ ratio & 5.975 & 0.015 & 1.158 & $1.028-1.304$ \\
\hline Age & 3.395 & 0.066 & 1.029 & 0.998-1.06 \\
\hline$\% \mathrm{PVO}_{2}$ & 3.385 & 0.064 & 0.992 & $0.985-1.00$ \\
\hline Creatinine mg/dL & 2.961 & 0.083 & 1.278 & $0.968-1.687$ \\
\hline Body mass index & 1.306 & 0.253 & 0.975 & $0.934-1.018$ \\
\hline Sodium mmol/L & 0.995 & 0.318 & 0.977 & $0.934-1.022$ \\
\hline Beta-blockers & 0.498 & 0.481 & 1.123 & $0.814-1.549$ \\
\hline Atrial fibrillation & 0.285 & 0.594 & 1.195 & $0.622-2.294$ \\
\hline Female gender & 0.139 & 0.709 & 0.934 & $0.654-1.335$ \\
\hline Ischemic etiology & 0.004 & 0.952 & 1.01 & $0.737-1.384$ \\
\hline
\end{tabular}

predictors of survival. Remarkably, in these studies EOB was not included in the final analysis.

In the present series, we could confirm that all-cause mortality for HF patients over 65 years is high, even in a stable outpatient population. By univariable analysis, common prognostic indicators such as age, NYHA class, LVEF, BNP, hemoglobin, a HF admission in the previous 12 months as well as most CPX variables, were associated to both mortality and HF readmissions (Tables 2 and 3 ). However, only the ventilatory variables $\mathrm{EOB}, \mathrm{VE} / \mathrm{VCO}_{2}$ slope and its ratio to $\mathrm{PVO}_{2}$, together with parameters indicative of clinical deterioration (hemoglobin, creatinine, and body mass index) retained an independent prognostic validity by multivariable analysis, independent of the level of systolic function. $\mathrm{PVO}_{2}$ per se was not independently predictive either of all-cause mortality or of the combined end-point. Conversely among CPX variables, a number of previous reports found that VE/ $\mathrm{VCO}_{2}$ slope $/ \mathrm{VO}_{2}$ and $\mathrm{EOB}$ are better prognostic predictors than $\mathrm{PVO}_{2}$ $[6,7,11,14]$.

The proposed pathogenetic mechanisms of EOB are multiple and include low cardiac output and the consequent increase in lung to chemoreceptor circulation time, a delayed response in the negative feedback loop that modulates ventilation and blood gas tension; impaired ventilatory control resulting in excessive ventilation due to an increased chemoreceptor sensitivity and/or to elevated LV filling pressure; and interstitial pulmonary edema with stimulation of vagal irritant receptors [7-9]. Data on the prevalence and quantification of EOB across various HF populations are not conclusive. In previous studies in middle-aged male patient cohorts $[7-9,14]$ the prevalence of EOB ranged from $12 \%$ to $35 \%$. In the only study conducted in

Table 4

Independent predictors of outcome by multivariable analysis.

\begin{tabular}{|c|c|c|c|c|}
\hline & Chi-square & $\mathrm{P}$ & HR & $95 \% \mathrm{CI}$ \\
\hline \multicolumn{5}{|l|}{ All-cause death } \\
\hline Hemoglobin & 24.898 & 0.0001 & 0.640 & $0.538-0.763$ \\
\hline $\mathrm{VE} / \mathrm{VCO}_{2}$ slope $/ \mathrm{PVO}_{2}$ & 10.180 & 0.001 & 1.269 & $1.096-1.468$ \\
\hline EOB & 8.987 & 0.003 & 2.641 & $1.400-4.983$ \\
\hline Creatinine & 7.707 & 0.005 & 1.725 & $1.174-2.535$ \\
\hline Body mass index & 4.403 & 0.036 & 0.918 & $0.887-0.984$ \\
\hline \multicolumn{5}{|l|}{ All-cause death + HF admissions } \\
\hline HF admission previous 12 months & 12.503 & 0.001 & 2.281 & $1.441-3.603$ \\
\hline Hemoglobin & 7.621 & 0.006 & 0.832 & $0.331-0.948$ \\
\hline $\mathrm{EOB}$ & 7.016 & 0.008 & 1.772 & $1.160-2.707$ \\
\hline $\mathrm{VE} / \mathrm{VCO}_{2}$ slope & 6.600 & 0.01 & 1.027 & $1.006-1.049$ \\
\hline
\end{tabular}




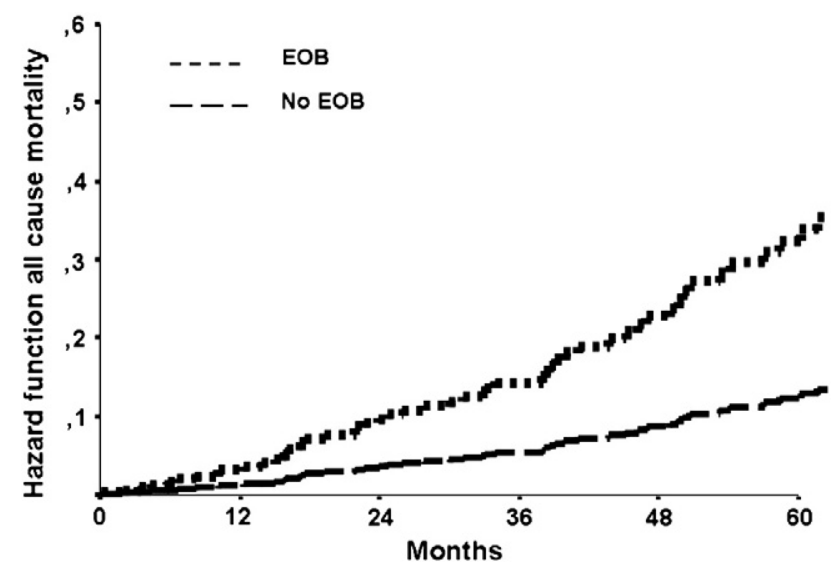

Fig. 1. Hazard function for all-cause mortality, adjusted by the other independent predictors of outcome, according to presence or absence of exercise oscillatory breathing (EOB).

patients with diastolic HF [14], the prevalence of EOB (31\%) was similar to the one observed in subjects with systolic dysfunction.

The prevalence of EOB has never been explored in elderly HF patients. Our study is the first to assess this phenomenon in a "real world" elderly HF population, as demonstrated by the relatively high prevalence of female gender and high median LVEF. We observed EOB in a proportion of patients (58\%) near double compared with the prevalence in younger subjects, potential heart transplant candidates [7-9,14]. Although we cannot fully explain the reasons for this high EOB prevalence in the elderly, the loss of elastic recoil of the chest wall due to the aging process might substantially contribute to the exacerbation of an oscillatory breathing pattern during exercise.

\subsection{Clinical implications}

The coexistence of EOB and ventilatory inefficiency identifies a population with severe circulatory and ventilatory instability, that carries a heavier burden of adverse events than conferred by any single ventilatory abnormality. Medical management for these patients should be reviewed and optimized whenever possible: beta-blockade [20] as well as ACE-inhibitors [21] has been shown to significantly reduce the $\mathrm{VE} / \mathrm{VCO}_{2}$ slope, while their effects on $\mathrm{EOB}$ are not known; tailored up-titration should also be considered. Aerobic

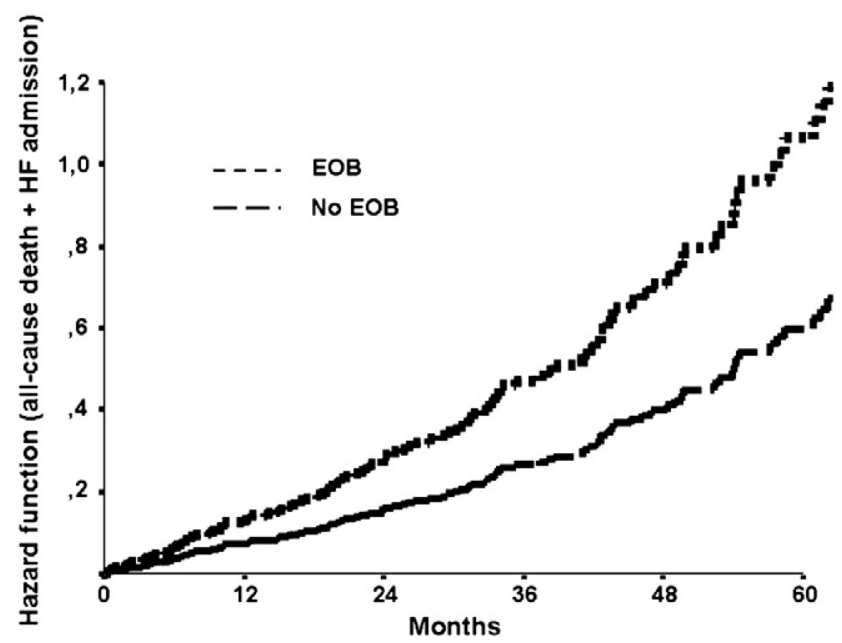

Fig. 2. Hazard function for the combined end point of all-cause mortality and admission for heart failure, adjusted by the other independent predictors of outcome, according to presence or absence of exercise oscillatory breathing (EOB).

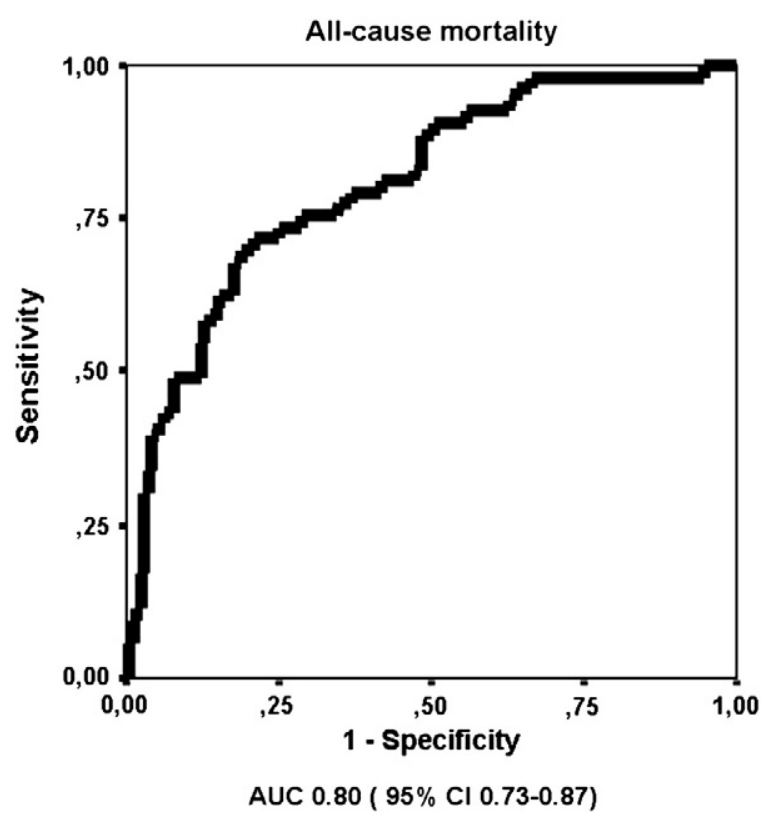

Fig. 3. ROC curve of the multivariable Cox model for all-cause mortality (AUC, area under the curve; CI confidence interval).

exercise training has likewise the potential to improve prognosis by reducing $\mathrm{VE} / \mathrm{VCO}_{2}$ slope [22]. Strict clinical follow-up should be envisaged to recognize early clinical signs of impending destabilization and to institute appropriate changes in treatment. Cardiac resynchronization therapy has been shown to significantly reduce the $\mathrm{VE} / \mathrm{VCO}_{2}$ slope and should be considered for high risk subjects, after optimized medical management.

\subsection{Study limitations}

Our study population was relatively small and included only clinically stable "robust" outpatients with mild to moderate HF, who did not have important comorbidities and were furthermore able to perform a CPX.

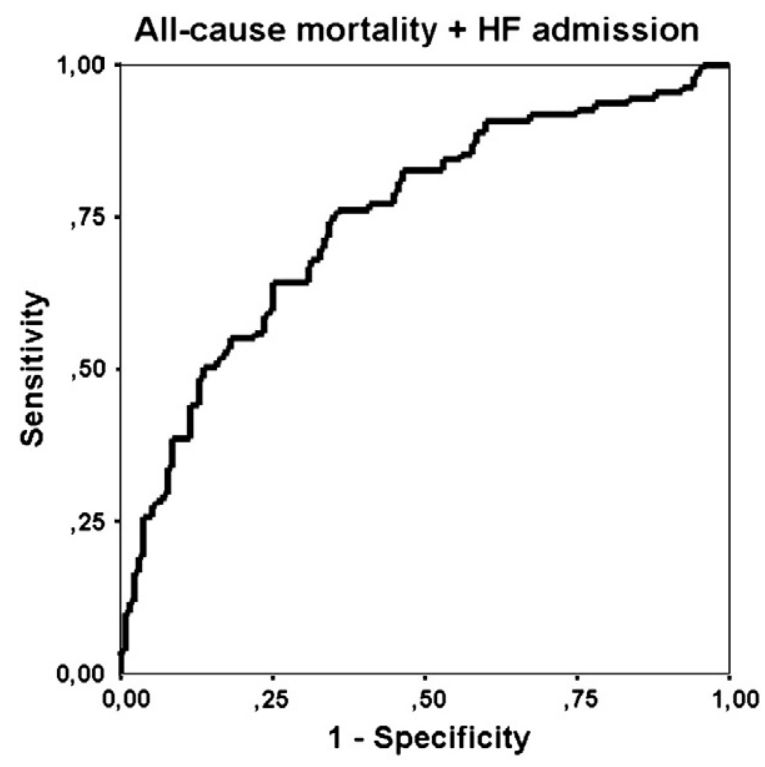

AUC 0.75 ( $95 \% \mathrm{Cl} 0.69-0.81)$

Fig. 4. ROC curve of the multivariable Cox model for all-cause mortality and admission for heart failure (AUC, area under the curve; $\mathrm{CI}$ confidence interval). 
Our study does not add any information on the pathophysiology that triggers and sustains EOB in elderly HF population. Nonetheless, the observed high EOB prevalence suggests that further studies are desirable in order to quantify EOB relevance in HF populations with more advanced disease stages.

We did not perform serial CPX to investigate whether some patients developed EOB during the follow-up and to assess the impact of optimized treatment on it. This is certainly an important point to address in future investigations.

In conclusion, an EOB pattern is remarkably frequent in elderly HF patients and represents a key marker of adverse outcome. These findings prospect the utility of comprehensively assessing CPXderived information to optimize the clinical and prognostic workup of elderly HF patients.

\section{Acknowledgement}

The authors do not hold any relationship with industry. The authors of this manuscript have certified that they comply with the Principles of Ethical Publishing in the International Journal of Cardiology [23].

\section{References}

[1] Task Force for Diagnosis and Treatment of Acute and Chronic Heart Failure 2008 of European Society of Cardiology. ESC Guidelines for the diagnosis and treatment of acute and chronic heart failure 2008: the Task Force for the Diagnosis and Treatment of Acute and Chronic Heart Failure 2008 of the European Society of Cardiology. Eur Heart J 2008;29:2388-442.

[2] Lund LH, Mancini DM. Peak $\mathrm{VO}_{2}$ in elderly patients with heart failure. Int J Cardiol 2008;125:166-71.

[3] Ingle L. Prognostic value and diagnostic potential of cardiopulmonary exercise testing in patients with chronic heart failure. Eur J Heart Fail 2008;10:112-8.

[4] Gibbons RJ, Balady GJ, Bricker JT, et al. American College of Cardiology/American Heart Association Task Force on Practice Guidelines. Committee to Update the 1997 Exercise Testing Guidelines. ACC/AHA 2002 guideline update for exercise testing: summary article. A report of the American College of Cardiology/American Heart Association Task Force on Practice Guidelines (Committee to Update the 1997 Exercise Testing Guidelines). J Am Coll Cardiol 2002;40:1531-40.

[5] Mezzani A, Agostoni P, Cohen-Solal A, et al. Standards for the use of cardiopulmonary exercise testing for the functional evaluation of cardiac patients. Eur J Cardiovasc Prev Rehabil 2009;6:249-67.

[6] Scardovi AB, Coletta C, De Maria R, et al. The cardiopulmonary exercise test is safe and reliable in elderly patients with chronic heart failure. J Cardiovasc Med (Hagerstown) 2007;8:608-12.
[7] Arena R, Myers J, Abella J, et al. Development of a ventilatory classification system in patients with heart failure. Circulation 2007;115:2410-7.

[8] Guazzi M, Arena R, Ascione A, Piepoli M, Guazzi MD. Exercise oscillatory breathing and increased ventilation to carbon dioxide production slope in heart failure: an unfavorable combination with high prognostic value. Am Heart J 2007;153: 859-67.

[9] Corrà U, Pistono M, Mezzani A, et al. Sleep and exertional periodic breathing in chronic heart failure: prognostic importance and interdependence. Circulation 2006;113:44-50.

[10] Guazzi M, Raimondo R, Vicenzi M, et al. Exercise oscillatory ventilation may predict sudden cardiac death in heart failure patients. J Am Coll Cardiol 2007;50: 299-308.

[11] Arena R, Myers J, Aslam SS, Varughese EB, Peberdy MA. Technical considerations related to the minute ventilation/carbon dioxide output slope in patients with heart failure. Chest 2003;124:720-7.

[12] Guazzi M, De Vita S, Cardano P, Barlera S, Guazzi MD. Normalization for peak oxygen uptake increases the prognostic power of the ventilatory response to exercise in patients with chronic heart failure. Am Heart J 2003;146:542-8.

[13] Leite JJ, Mansur AJ, de Freitas HF, et al. Periodic breathing during incremental exercise predicts mortality in patients with chronic heart failure evaluated for cardiac transplantation. J Am Coll Cardiol 2003;41:2175-8.

[14] Garcia MJ, Thomas JD, Klein AL. New Doppler echocardiographic applications for the study of diastolic function. J Am Coll Cardiol 1998;32:865-75.

[15] Guazzi M, Myers J, Peberdy MA, Bensimhon D, Chase P, Arena R. Exercise oscillatory breathing in diastolic heart failure: prevalence and prognostic insights. Eur Heart J 2008;29:2751-9.

[16] Davies LC, Francis DP, Piepoli M, Scott AC, Ponikowski P, Coats AJ. Chronic heart failure in the elderly: value of cardiopulmonary exercise testing in risk stratification. Heart 2000;83:147-51.

[17] Mejhert M, Linder-Klingsell E, Edner M, Kahan T, Persson H. Ventilatory variables are strong prognostic markers in elderly patients with heart failure. Heart 2002;88:239-43.

[18] Cicoira M, Davos CH, Florea V, et al. Chronic heart failure in the very elderly: clinical status, survival and prognostic factors in 188 patients more than 70 years old. Am Heart J 2001;142:174-80.

[19] Scardovi AB, De Maria R, Celestini A et al Additive prognostic value of cardiopulmonary exercise testing in elderly patients with heart failure. Clin Sci (Lond) 2009;116:415-22.

[20] Parikh MN, Lund LH, Goda A, Mancini D. Usefulness of peak exercise oxygen consumption and the heart failure survival score to predict survival in patients $>65$ years of age with heart failure. Am J Cardiol 2009;103:998-1002.

[21] Agostoni P, Guazzi M, Bussotti M, De Vita S, Palermo P. Carvedilol reduces the inappropriate increase of ventilation during exercise in heart failure patients. Chest 2002;122:2062-7.

[22] Guazzi M, Marenzi G, Alimento M, Contini M, Agostoni P. Improvement of alveolar-capillary membrane diffusing capacity with enalapril in chronic heart failure and counteracting effect of aspirin. Circulation 1997:95:1930-6.

[23] Shewan LG, Coats AJ. Ethics in the authorship and publishing of scientific articles. Int J Cardiol 2010;144:1-2 